\title{
Velocity scaling for filament motion in scrape-off layer plasmas
}

\author{
R. Kube and O. E. Garcia ${ }^{\text {a) }}$ \\ University of Troms $\phi$, Department of Physics and Technology, $N 9037$ Troms $\phi$, Norway
}

(Received 28 June 2011; accepted 14 September 2011; published online 21 October 2011)

\begin{abstract}
The velocity scaling for isolated plasma filaments in non-uniformly magnetized plasmas with respect to filament amplitude and cross-field size has been investigated by means of numerical simulations. The model includes electric currents due to magnetic gradient and curvature drifts, polarization drifts, and parallel currents through sheaths, where the magnetic field lines intersect material walls. In the ideal limit, the radial velocity of the filament increases with the square root of its size. When sheath currents dominate over polarization currents, the filament velocity is inversely proportional to the square of its size. In the presence of sheath currents, the velocity is maximum for an intermediate filament size determined by the balance between diamagnetic, polarization, and sheath currents. The parameter dependence of this filament size and velocity is elucidated. The results are discussed in the context of blob-like structures in basic laboratory plasma experiments and in the scrape-off layer of magnetically confined plasmas. (C) 2011 American Institute of Physics. [doi:10.1063/1.3647553]
\end{abstract}

\section{INTRODUCTION}

Transport of particles and heat across magnetic field lines in the scrape-off layer of magnetically confined plasmas has been found to be dominated by radial motion of filamentary structures. These structures are elongated along the magnetic field and localized in the drift plane perpendicular to the field lines, thus appearing as blobs of excess particles and heat compared to the background plasma. ${ }^{1-5}$

Much attention has been given to the dynamics of localized filamentary structures in magnetized plasmas. Probe measurements in the scrape-off layer of tokamak plasmas have revealed frequent appearance of large-amplitude bursts which give rise to positively skewed and flattened particle density probability distribution functions and conditionally averaged wave forms with a steep front and a trailing wake. ${ }^{6-12}$ Fast camera imaging in numerous experiments have demonstrated radial motion of blob-like structures through the scrape-off layer with velocities up to one tenth of the acoustic speed. ${ }^{13-17}$

Theoretical modelling and numerical simulations of isolated filament structures have revealed interchange dynamics in the non-uniform magnetic field as the likely mechanism for the radial filament motion. ${ }^{18-23}$ Two limits for the parallel dynamics have been identified. In the absence of parallel currents, the radial filament velocity is found to increase as the square root of its cross-field size. ${ }^{19,20}$ However, when diamagnetic currents are balanced by parallel currents through sheaths where the magnetic field lines intersect material walls, the filament velocity is inversely proportional to the square of its size. ${ }^{20-22}$ In this contribution, we reveal the velocity dependence of the filament on its size and amplitude in the intermediate regime between these two extremes.

Recently, several experiments on filament dynamics have been made in basic toroidal laboratory plasmas. In the case of

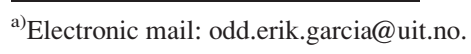

a purely toroidal magnetic field in the Versatile Toroidal Facility (VTF) device, an isolated plasma filament was produced at the high field side and allowed to evolve freely. ${ }^{24}$ In these experiments, it was found that large-amplitude filaments can move radially outwards with velocities comparable to the acoustic speed. ${ }^{24}$ The spatio-temporal evolution is qualitatively similar to results from numerical simulations of blob dynamics in a non-uniform magnetic field. ${ }^{19,20}$ However, the filaments in these experiments have very large amplitudes while the simulation results were restricted to small relative amplitudes. Here, we generalize the previous theories to obtain the velocity dependence for large filament amplitudes.

In other basic plasma torus experiments on the TORPEX device, a metal limiter introduced on the low-field side resulted in parallel electric currents through sheaths where the magnetic field lines intersect material walls. ${ }^{25}$ Blob-like structures were found to develop from interchange turbulence and their radial motion influenced by sheath currents to the limiter plates. ${ }^{25}$ The results presented here are in excellent agreement with the blob size dependence of the velocity found in these experiments. This configuration resembles the scrape-off layer in magnetically confined plasmas.

This paper is organized as follows. In Sec. II, we present the model equations, the normalization used, and the asymptotic velocity scalings for isolated plasma filaments. In Sec. III, we present results from numerical simulations of filament dynamics with varying filament amplitude and sheath dissipation parameter. These results are discussed in Sec. IV, with emphasis on the filament velocity dependence on the cross-field size and amplitude. A summary and conclusions of this work is given in Sec. V.

\section{INTERCHANGE MODEL}

The radial motion of filament structures in the scrapeoff layer of tokamak plasmas is believed to be caused by the charge polarization within the structures due to vertical magnetic field gradient and curvature drifts. ${ }^{1-5,9-12,18-23}$ This 
current may be closed by polarization drifts or by parallel currents through sheaths where the magnetic field lines intersect material walls. The cross-field size and amplitude of the plasma filament determines the relative magnitude of these current channels and, thereby, the radial filament velocity.

Similar to most previous studies of filament motion in non-uniformly magnetized plasmas, we use a two-field model describing the evolution of the particle density $n$ and electric drift vorticity $\Omega=\mathbf{b} \cdot \nabla \times(\mathbf{b} \times \nabla \phi / B)=\nabla_{\perp}^{2} \phi / B$ (Refs. 2 and 20),

$$
\begin{gathered}
\left(\frac{\partial}{\partial t}+\frac{1}{B} \mathbf{b} \times \nabla \phi \cdot \nabla\right) n=\kappa_{e} \nabla_{\perp}^{2} n, \\
\left(\frac{\partial}{\partial t}+\frac{1}{B} \mathbf{b} \times \nabla \phi \cdot \nabla\right) \Omega+C_{s}^{2} \mathbf{b} \\
\times(\mathbf{b} \cdot \nabla \mathbf{b}+\nabla \ln B) \cdot \nabla \ln n=\mu_{i} \nabla_{\perp}^{2} \Omega+\sigma \frac{\phi}{B},
\end{gathered}
$$

where $C_{s}$ is the sound speed, $\phi$ is the electrostatic potential, b is the unit vector along the magnetic field, $\sigma=C_{s} / L_{\|} \rho_{s}^{2}$ is the sheath dissipation parameter due to parallel electric currents through sheaths at material surfaces, $L_{\|}$is the magnetic connection length, $\rho_{s}$ is the sound gyration radius, and $\kappa_{e}$ and $\mu_{i}$ are the collisional particle diffusion and viscosity coefficients, respectively. In deriving the sheath dissipation term in Eq. (1b), the filament structure is assumed to be uniform along the magnetic field lines. ${ }^{20}$

We introduce a local coordinate system with $x$ in the radial direction, $y$ in the poloidal direction, and $z$ along the magnetic field. Spatial scales are normalized with a characteristic cross-field filament size $\ell$ and temporal scales with the corresponding ideal interchange rate $\gamma_{0}=(g / \ell)^{1 / 2}$, where $g=2 C_{s}^{2} / R$ is the effective gravity and $R$ is the magnetic field length scale. In the following, we will use these non-dimensional units unless otherwise explicitly stated. The non-dimensional model is then written in the form,

$$
\begin{gathered}
\left(\frac{\partial}{\partial t}+\hat{\mathbf{z}} \times \nabla \phi \cdot \nabla\right) \ln n=\kappa \nabla_{\perp}^{2} \ln n-\kappa\left(\nabla_{\perp} \ln n\right)^{2}, \\
\left(\frac{\partial}{\partial t}+\hat{\mathbf{z}} \times \nabla \phi \cdot \nabla\right) \Omega+\frac{\partial \ln n}{\partial y}=\mu \nabla_{\perp}^{2} \Omega+\Lambda \phi,
\end{gathered}
$$

where the non-dimensional sheath dissipation coefficient is given by $\Lambda=\left(C_{s} \ell^{2} / \gamma_{0} L_{\|} \rho_{s}^{2}\right)$ and the non-dimensional diffusion coefficients are given by $\kappa=\kappa_{e} / \gamma_{0} \ell^{2}$ and $\mu=\mu_{i} / \gamma_{0} \ell^{2}$.

Currents due to particle drifts in the non-uniform magnetic field, described by the last term on the left hand side of Eq. (2b), give rise to polarization of vorticity across the field lines and thus a radial electric drift at the centre of a bloblike structure. ${ }^{18-20}$ Sheath currents, described by the last term on the right hand side of Eq. (2b), give rise to damping of collective motions, preferentially on large spatial length scales. $^{20}$

In the following section, we present results from numerical simulations of the model (2). The initial condition for the simulations is a localized, blob-like structure for the particle density,

$$
\frac{n}{N}=1+\frac{\Delta n}{N} \theta
$$

where $N$ is the background particle density, $\Delta n / N$ is the relative filament amplitude, and the spatial variation is given by $\theta$ which initially is taken to be $\mathrm{e}^{19,20}$

$$
\theta(\mathbf{x}, t=0)=\exp \left(-\frac{1}{2} \mathbf{x}^{2}\right) .
$$

There is no initial fluid flow, $\phi(\mathbf{x}, t=0)=0$, so the collective motions arise from charge polarization due to particle drifts in the non-uniform magnetic field, which is described by the interchange term in the vorticity equation $(2 b)$.

In the small filament amplitude limit, $\Delta n / N \ll 1$, the interchange term in the vorticity equation ( $2 b)$ can be linearised, $\partial \ln n / \partial y \sim \Delta n / N$. In the ideal limit, $\Lambda \ll 1$, a simple order of magnitude estimate gives the inertial velocity scaling, $V^{2} \sim \Delta n / N$, which in dimensional units is given by ${ }^{19,20}$

$$
\frac{V}{C_{s}} \sim\left(\frac{2 \ell}{R} \frac{\Delta n}{N}\right)^{1 / 2} .
$$

In this limit, the filament velocity increases as the square root of the cross-field size $\ell$ and the relative amplitude. However, for large $\Delta n / N$ the interchange term is independent of the amplitude, $\partial \ln n / \partial y \sim \Delta n /(N+\Delta n) \sim 1$, so the velocity is then expected to become independent of the filament amplitude. Further discussions of the filament velocity dependence on the amplitude will be given in the following two sections.

When sheath dissipation dominates, $\Lambda \gg 1$, an order of magnitude estimate for large filament amplitudes give the velocity scaling $V \sim 1 / \Lambda$, which in dimensional units is given by ${ }^{20-22}$

$$
\frac{V}{C_{s}} \sim \frac{2 L_{\|} \rho_{s}^{2}}{R \ell^{2}} .
$$

This predicts a velocity that is inversely proportional to the square of the cross-field filament size. Neglecting collisional diffusion and viscosity, the model (2) has a well-known analytical solution given by ${ }^{20-22}$

$$
\begin{gathered}
\phi=-\frac{y}{\Lambda}, \\
\frac{n}{\Delta n}=\Theta\left(x-\frac{t}{\Lambda}\right) \exp \left(-\frac{y^{2}}{2}\right) .
\end{gathered}
$$

This describes a blob-like structure with arbitrary radial variation, initially given by $\Theta(x)$, moving radially outwards with velocity $1 / \Lambda$. It should be noted that according to this solution, the plasma filament moves in vacuum, $N=0$, with no associated vorticity, $\Omega=0$.

The interchange model (2) predicts that the filament velocity increases with size for small $\ell$ and decreases with size for large $\ell$. It follows that sheath-connected filaments have a maximum velocity for an intermediate cross-field filament size determined by the balance between the diamagnetic, polarization, and sheath currents. An order of magnitude estimate of this length scale is given by the sheath dissipation parameter $\Lambda=\left(\ell / \ell_{*}\right)^{5 / 2}$ equal to unity, which gives ${ }^{26-28}$ 

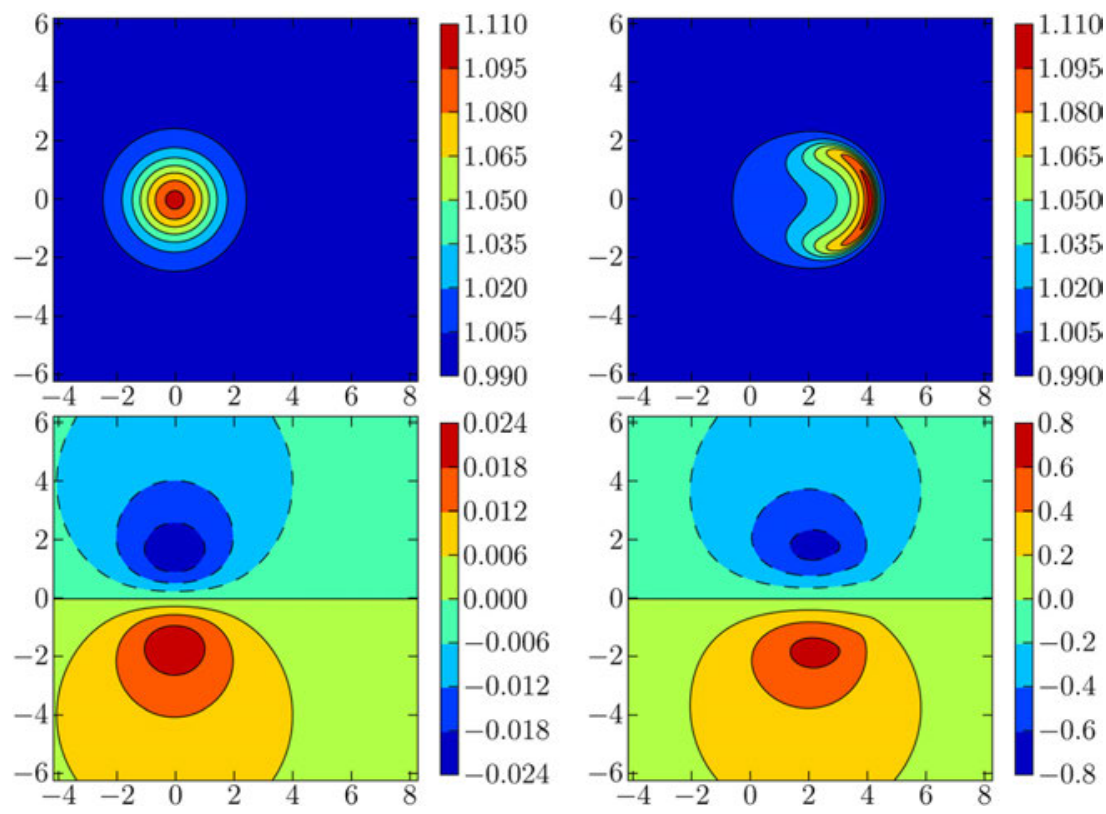

FIG. 1. (Color online) Particle density (top row) and electrostatic potential (bottom row) at $t \quad 0.5$ (left column) and $t \quad 16$ (right column) for $\Delta n / N \quad 10$ and $\Lambda \quad 0$. Only a small part of the simulation do main is shown.

$$
\ell_{*}=\left(\frac{2 \rho_{s}^{4} L_{\|}^{2}}{R}\right)^{1 / 5} .
$$

The magnitude of the maximum filament velocity in dimensional units is estimated by Eq. (5) for this filament size, $V_{*} / C_{s}=\left(2 \ell_{*} / R\right)^{1 / 2}$, or in terms of the plasma parameters, ${ }^{26-28}$

$$
\frac{V_{*}}{C_{s}}=\left(\frac{8 \rho_{s}^{2} L_{\|}}{R^{3}}\right)^{1 / 5} .
$$

In Sec. IV, the prefactors for this size and velocity scaling and their amplitude dependence will be established based on results from numerical simulations presented in Sec. III.

\section{NUMERICAL SIMULATIONS}

In this section, we present results from numerical simulations of the interchange model (2). The model is solved on a biperiodic domain using a standard Fourier Galerkin method. The normalized diffusion coefficients $\kappa$ and $\mu$ are both set to $10^{-3}$, which ensures that we are in the ideal regime even for relative filament amplitudes as small as $10^{-2}$ in the absence of sheath dissipation. ${ }^{19,20}$ Numerical simulations have been done for relative filament amplitudes $\Delta n / N$ from $10^{-2}$ to $10^{5}$ and the sheath dissipation parameter $\Lambda$ from zero to $10^{2}$. Convergence with respect to spatial and temporal resolution and the size of the simulation domain has been carefully checked for all simulations. A square simulation domain has been used with normalized box length up to 100 and number of grid points up to $8192^{2}$.

In order to quantify the motion of the plasma filament, we define the radial centre of mass position and velocity,

$$
\begin{gathered}
X(t)=\int d \mathbf{x} x \theta / \int d \mathbf{x} \theta, \\
V(t)=\frac{d X}{d t},
\end{gathered}
$$

where the integration is over the whole simulation domain and $\theta$ is defined in Eq. (3). Multiplying the particle density equation (2a) by $x$ and integrating over space, it follows that the radial centre of mass velocity is equal to the total particle flux due to collective motions,

$$
V(t)=-\int d \mathbf{x} \theta \frac{\partial \phi}{\partial y} / \int d \mathbf{x} \theta
$$

The centre of mass velocity is thus also a measure of the radial particle transport caused by the motion of the filament structure.

\section{A. Inertial regime}

We first present results from numerical simulations where the blob amplitude has been varied and sheath dissipation has been neglected, $\Lambda=0$. The inertial response to vertical gradients in the particle density causes the formation of a vorticity dipole structure and, hence, a radially outwards electric drift at the centre of the filament structure. This leads to the formation of a steep front and a trailing wake, as shown by the contour plots in Fig. 1 and the radial variation of the particle density at the symmetry plane $y=0$ presented in Fig. 2 for $\Delta n / N=10^{-1}$. The evolution of the

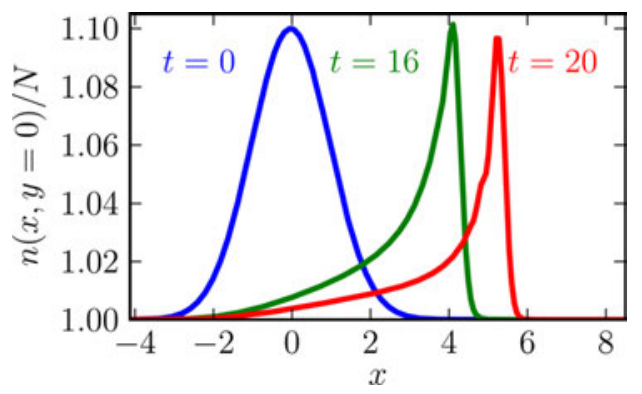

FIG. 2. (Color online) Radial variation of the particle density at $y \quad 0$ for various times and $\Delta n / N \quad 10^{1}$ and $\Lambda \quad 0$. 


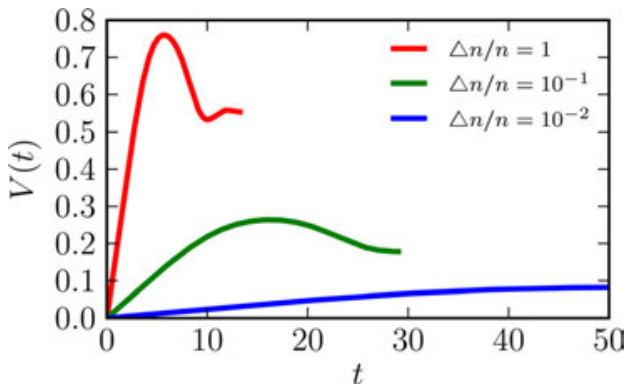

FIG. 3. (Color online) Evolution of the radial centre of mass velocity for $\Lambda \quad 0$.

radial centre of mass velocity, presented in Fig. 3, shows that there is an initial acceleration and subsequent deceleration, resulting in a pronounced peak of the velocity curve. The time of maximum radial centre of mass velocity is $t=16$ for the case $\Delta n / N=10^{-1}$. The filaments radial centre of mass coordinate is then $X(16)=2.7$, that is, the filament has moved a radial distance nearly three times its initial size. By then it has developed the shape of a mushroom-like cap, so the interchange mechanism leads to small-scale flows and dispersion of the filament structure. The subsequent evolution depends sensitively on the diffusion parameters. ${ }^{20}$

In Fig. 3, we compare the evolution of the radial centre of mass velocity for various filament amplitudes. For all $\Delta n / N$, the velocity curve has a pronounced peak. The time at which the filament has maximum velocity scales as $(\Delta n / N)^{1 / 2}$ for relative amplitudes smaller than unity, in agreement with previous work for small amplitude filaments. ${ }^{19,20}$ The filament dynamics for large amplitudes is qualitatively similar to that for small $\Delta n / N$. However, from the contour plots presented in Fig. 4 for $\Delta n / N=10^{2}$, it is seen that the filament retains its blob-like structure at the time of maximum radial centre of mass velocity, which is at $t=2$ in this case. The radial centre of mass coordinate for the filament is then $X(2)=3.3$.

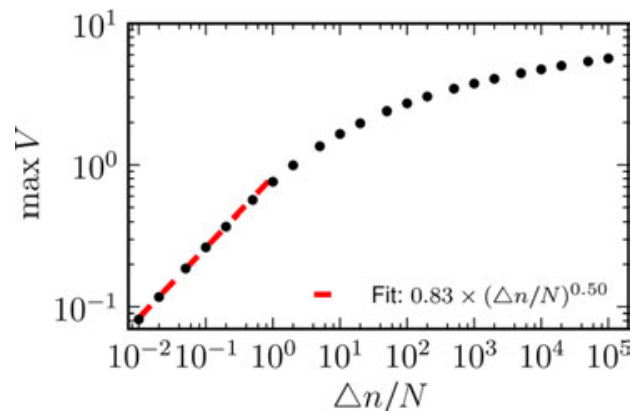

FIG. 5. (Color online) Maximum radial centre of mass velocity as a function of relative filament amplitude for $\Lambda \quad 0$. The dashed line is a least squares fit of a power law to the simulation data.

In Fig. 5, we present the maximum radial centre of mass velocity of the filament as a function of its relative amplitude over seven orders of magnitude. For $\Delta n / N<1$, we find that the filament velocity increases as the square root of its amplitude. A least squares fit gives $V=0.83(\Delta n / N)^{0.50}$, in excellent agreement with previous results for small amplitude filaments. ${ }^{19,20}$ For relative filament amplitudes larger than unity the square root scaling for the radial velocity clearly does not hold. There is a very weak dependence of the velocity on the amplitude for large $\Delta n / N$. However, the velocity is not seen to become independent of the amplitude as expected from the discussion at the end of Sec. II. This is likely due to the more persistent blob-like structure during the filament motion which sustains the dipole vorticity generation and, thereby, larger radial velocities compared to the low amplitude case.

\section{B. Sheath dissipation}

In this subsection, we present results from numerical simulations where the sheath dissipation parameter $\Lambda$ has been varied. This has been done for filament amplitudes
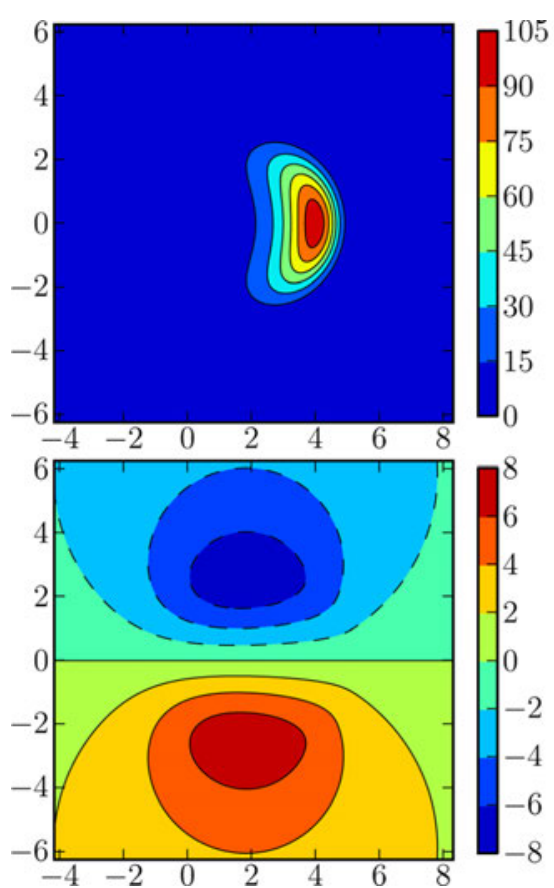
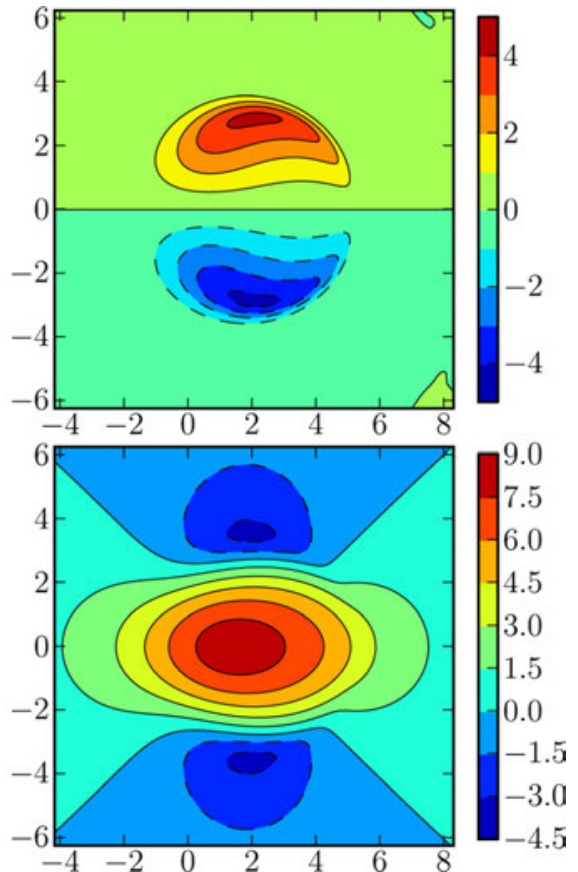

FIG. 4. (Color online) Structure of the particle density (top left), vorticity (top right), electro static potential (bottom left), and radial velocity (bottom right) for $\Delta n / N \quad 10^{2}$ and $\Lambda \quad 0$ at time $t \quad 2$. 


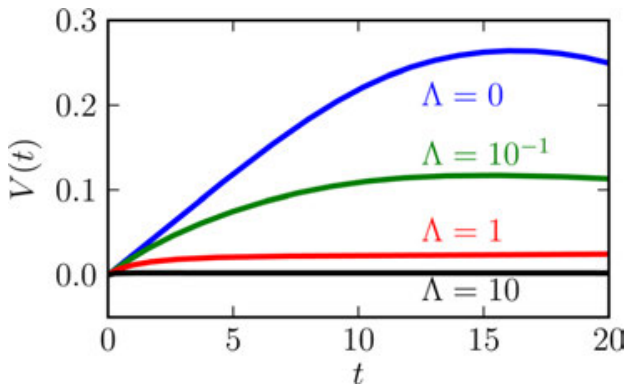

FIG. 6. (Color online) Evolution of the radial centre of mass velocity for $\Delta n / N \quad 10^{1}$ and various sheath dissipation parameters.

$\Delta n / N$ up to 100 . The most prominent effect of sheath currents on filament dynamics is that they reduce the radial velocity and, thereby, the cross-field plasma transport. In Fig. 6 , we present the evolution of the radial centre of mass velocity from simulations with $\Delta n / N=10^{-1}$ for different $\Lambda$. The maximum velocity decreases gradually with increasing sheath dissipation parameter. For small values of $\Lambda$ we find that the velocity curves feature a pronounced peak. For $\Lambda$ of order unity or larger, the velocity becomes approximately constant after a short period of acceleration. For $\Delta n / N=10^{-1}$ and $\Lambda=10$ the filament structure is effectively stagnant. In Fig. 7 , we present contour plots of the particle density, vorticity, electrostatic potential, and radial velocity for $\Lambda=10^{-1}$ at $t=15$, the time of maximum radial centre of mass velocity. Compared to Fig. 1, we see that the formation of a mushroom-like cap is less pronounced and the electrostatic potential has significantly smaller amplitude and spatial extent in the presence of sheath dissipation. The radial centre of mass position at the time of maximum velocity is $X(t=15)=1.27$, less than half of that for $\Lambda=0$. These results are similar to those presented previously for small amplitude filaments. ${ }^{20}$

The evolution of the radial centre of mass velocity for $\Delta n / N=10^{2}$ and various sheath dissipation parameters is

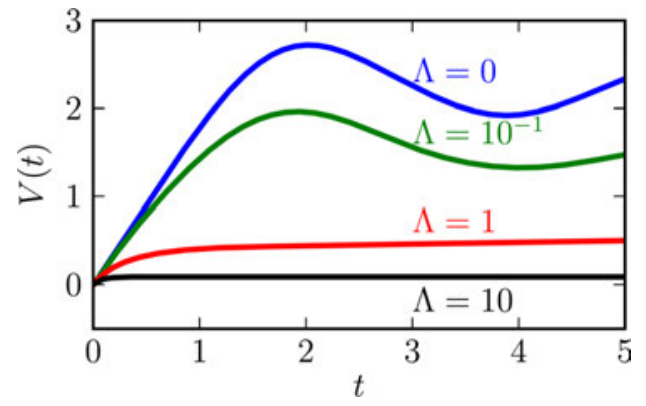

FIG. 8. (Color online) Evolution of the radial centre of mass velocity for $\Delta n / N \quad 10^{2}$ and various sheath dissipation parameters.

presented in Fig. 8. The maximum velocity clearly increases with increasing filament amplitude and decreasing sheath dissipation parameter. The filament evolution for large $\Delta n / N$ and small $\Lambda$ is qualitatively similar to that for $\Lambda=0$. Increasing the sheath dissipation parameter $\Lambda$ to unity or larger, we find that the radial centre of mass velocity is reduced substantially. As seen in Fig. 9 for $\Delta n / N=10^{2}$ and $\Lambda=10$, the filament structure keeps its blob-like shape while it is slowly moving radially outwards. The radial velocity is nearly constant at the centre of the structure. However, the radial centre of mass velocity is very small. Hence, the radial centre of mass position at $t=2$ is only 0.17 . It should be noted that for large $\Lambda$ and $\Delta n / N$ the structure from the numerical simulation resembles the analytical blob solution in Eq. (7) in the centre with zero vorticity and a Gaussian particle density.

In Fig. 10, we present the maximum radial centre of mass velocity as function of the relative filament amplitude for various sheath dissipation parameters. For each value of $\Lambda$, the maximum velocity scales as a power law with $\Delta n / N$ for relative amplitudes less than unity. Through a least squares fit in the range $0.1 \leq \Delta n / N \leq 1$, a scaling of the
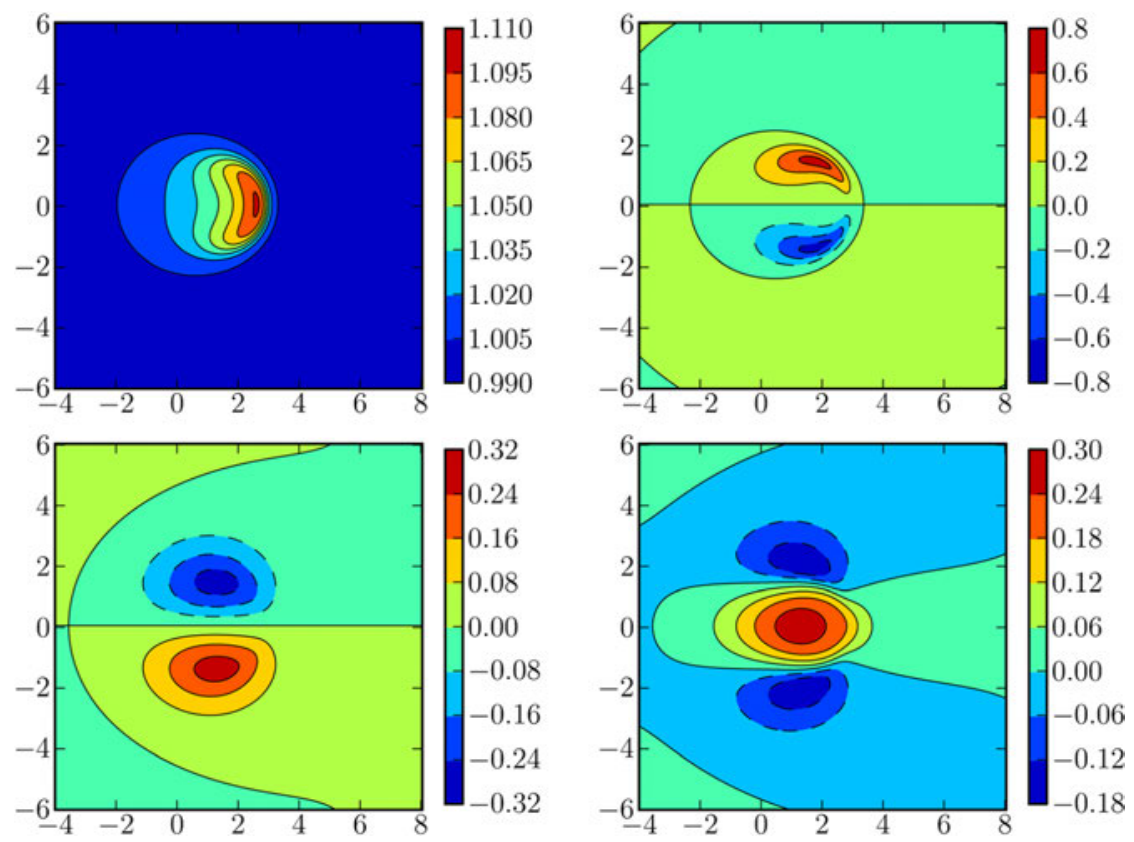

FIG. 7. (Color online) Structure of the particle den sity (top left), vorticity (top right), electrostatic potential (bottom left), and radial velocity (bottom right) for $\Delta n / N \quad 10^{1}$ and $\Lambda \quad 10^{1}$ at time $t \quad 15$. 

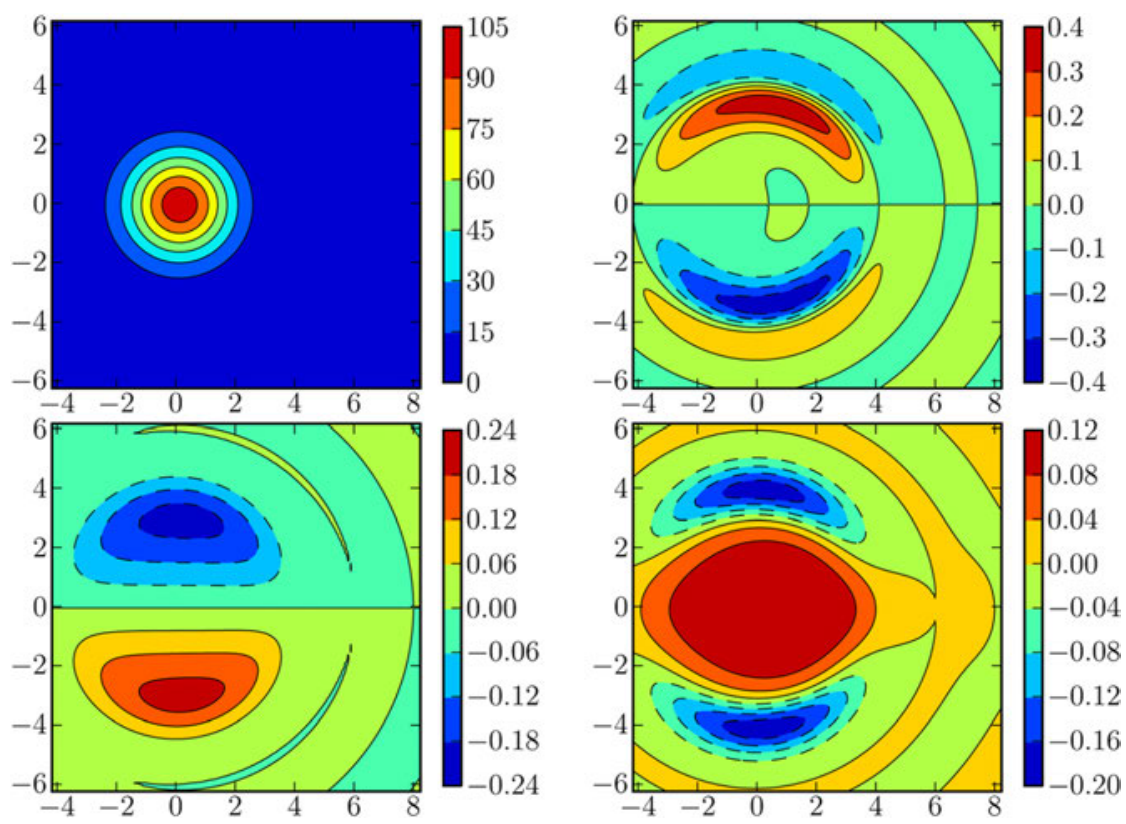

FIG. 9. (Color online) Structure of the particle den sity (top left), vorticity (top right), electrostatic potential (bottom left), and radial velocity (bottom right) for $\Delta n / N \quad 10^{2}$ and $\Lambda \quad 10$ at time $t \quad 2$.

maximum radial centre of mass velocity on the form $V \sim(\Delta n / N)^{\beta}$ is found for each $\Lambda$. The scaling exponent $\beta$ increases gradually from 0.50 for $\Lambda=0$ to approximately 0.85 for $\Lambda=1$ and does not increase further with increasing sheath dissipation parameter up to 100 . The change in the scaling exponent for $\Lambda$ approximately equal to unity comes along with a qualitative change in the evolution of the radial centre of mass velocity, which no longer features a pronounced maximum for $\Lambda>1$, as seen in Figs. 6 and 8. As $\Delta n / N$ approaches unity, the velocity scaling in Fig. 10 breaks and the maximum velocity increases only slightly with further increasing filament amplitude. This behaviour is similar to that seen in Fig. 5 and expected from the discussion at the end of Sec. II.

The variation of the maximum radial centre of mass velocity as function of the sheath dissipation parameter for various filament amplitudes is presented in Fig. 11. The simulation data shows that for fixed $\Delta n / N$, the maximum velocity is inversely proportional to $\Lambda$ for large sheath currents. Indeed, the velocity scaling from the analytical solution in

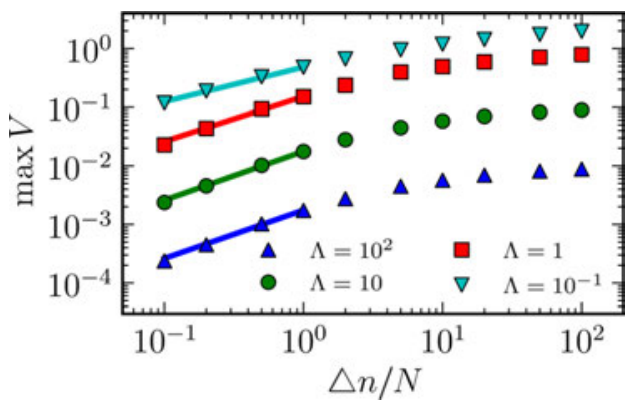

FIG. 10. (Color online) Maximum radial centre of mass velocity as a func tion of relative filament amplitude for various sheath dissipation parameters. The lines are least squares fits of a power law to the simulation data for small amplitudes.
Eq. (6), $1 / \Lambda$, appears to be the upper limit for the filament velocity. Note however that the velocity scaling from the analytical solution grossly over-estimates the velocity for either $\Lambda$ or $\Delta n / N$ of order unity or smaller.

\section{DISCUSSION}

Results from numerical simulations with varying filament amplitude and sheath dissipation parameter confirm that the filament velocity is proportional to the square root of the filament size $\ell$ when $\Lambda$ is small, and that the velocity is inversely proportional to the square of the filament size for large $\Lambda$. In this section, we present an order of magnitude estimate for the filament velocity and its size dependence and compare this to the simulation results.

A simple order of magnitude estimate of the various terms in the non-dimensional vorticity equation $(2 \mathrm{~b})$, with $\partial / \partial t \sim \Omega \sim V$, gives

$$
V^{2}-c_{1} \frac{\Delta n / N}{1+\Delta n / N}+c_{2} \Lambda V=0
$$

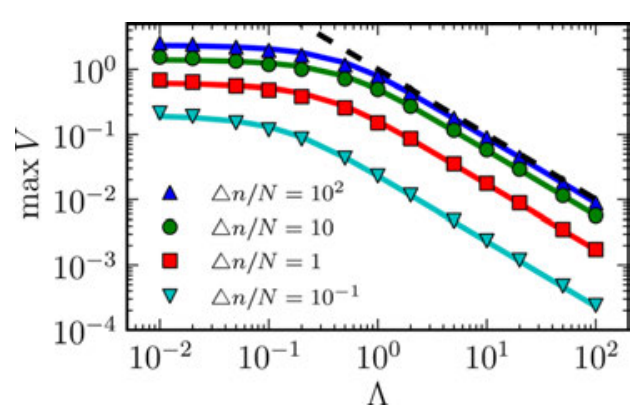

FIG. 11. (Color online) Maximum radial centre of mass velocity as a func tion of the sheath dissipation parameter $\Lambda$ for various relative filament amplitudes. The dashed line corresponds to $V \quad 1 / \Lambda$. The full lines are least squares fit of an order of magnitude approximation to simulation data. 


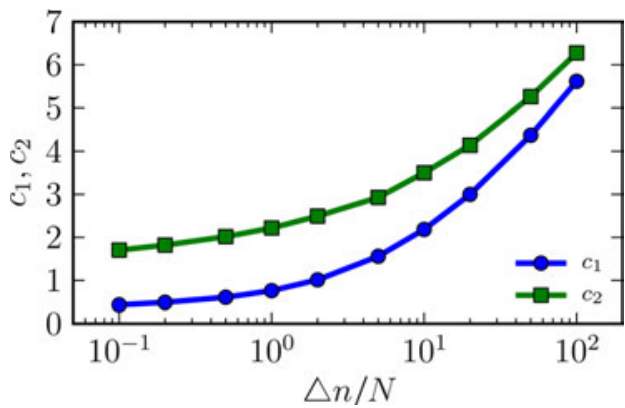

FIG. 12. (Color online) Fit coefficients $c_{1}$ and $c_{2}$ for parametrization of the maximum radial filament velocity as a function of the relative filament amplitude.

where $c_{1}$ and $c_{2}$ are fitting parameters that depend on the relative filament amplitude. The positive root for the filament velocity is given by

$$
V=-\frac{c_{2} \Lambda}{2}+\left(\frac{c_{2}^{2} \Lambda^{2}}{4}+c_{1} \frac{\Delta n / N}{1+\Delta n / N}\right)^{1 / 2} .
$$

By fitting the model (13) to the data from numerical simulations for fixed filament amplitude, we find the parameters $c_{1}$ and $c_{2}$. The fit to the simulation data is presented in Fig. 11 and the fit coefficients $c_{1}$ and $c_{2}$ as the function of the filament amplitude are shown in Fig. 12. Equation (13) is clearly a very good approximation to the simulation results.

The asymptotic solutions for $V$ based on Eq. (13) in the limit of small and large $\Lambda$ are given by

$$
V \sim \begin{cases}\left(c_{1} \frac{\Delta n / N}{1+\Delta n / N}\right)^{1 / 2}, & \text { for } \Lambda \ll 1, \\ \frac{c_{1}}{c_{2} \Lambda} \frac{\Delta n / N}{1+\Delta n / N}, & \text { for } \Lambda \gg 1 .\end{cases}
$$

These are just the ideal and sheath dissipative limits discussed in Sec. II, but here generalized to include a large range of filament amplitudes. Reverting to dimensional units, the size dependence of the filament velocity implied by Eq. (13) becomes explicit

$$
\frac{V}{V_{*}}=\frac{c_{2}}{2}\left(\frac{\ell}{\ell_{*}}\right)^{3}\left[-1+\left(1+\frac{c_{1}}{c_{2}^{2}} \frac{4 \ell_{*}^{5}}{\ell^{5}} \frac{\Delta n / N}{1+\Delta n / N}\right)^{1 / 2}\right] .
$$

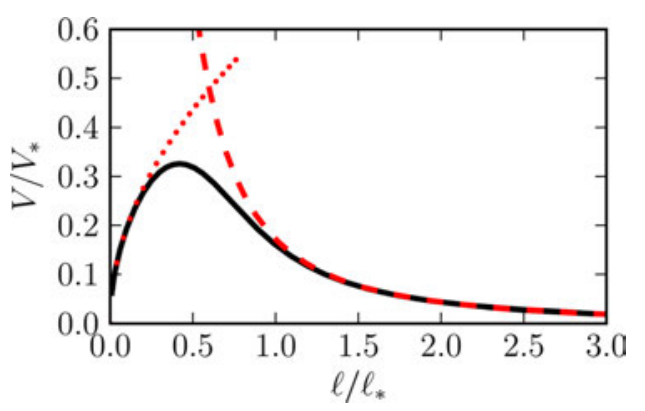

FIG. 13. (Color online) Dimensional filament velocity dependence on cross field size $\ell$ for $\Delta n / N \quad 1$ in the presence of sheath dissipation (full line). For $\ell / \ell_{*} \ll 1$, the inertial scaling gives $V \sim \ell^{1 / 2}$ (dotted line) while for $\ell / \ell_{*} \gg 1$, the sheath dissipative scaling gives $V \sim \ell^{2}$ (broken line).

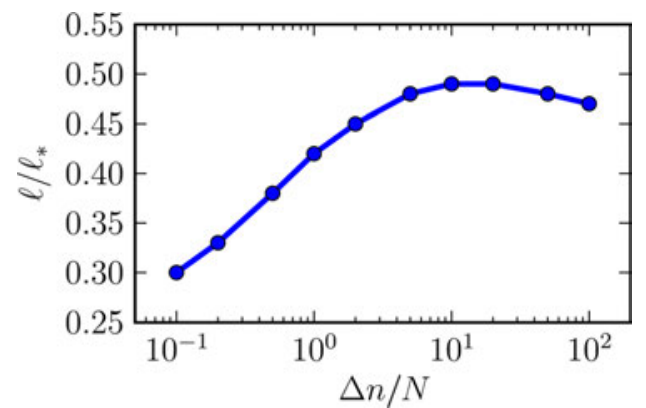

FIG. 14. (Color online) Size for which the radial filament velocity is maxi mum as a function of the relative filament amplitude in the presence of sheath dissipation.

For small filament sizes, $\ell \ll \ell_{*}$, we find

$$
\frac{V}{V_{*}} \sim\left(c_{1} \frac{\ell}{\ell_{*}} \frac{\Delta n / N}{1+\Delta n / N}\right)^{1 / 2}
$$

which for small filament amplitudes corresponds to the inertial scaling in Eq. (5). For large filament sizes, $\ell \gg \ell_{*}$, Eq. (15) gives

$$
\frac{V}{V_{*}} \sim \frac{c_{1}}{c_{2}}\left(\frac{\ell_{*}}{\ell}\right)^{2} \frac{\Delta n / N}{1+\Delta n / N},
$$

which in the limit of large filament amplitudes corresponds to the sheath dissipative scaling in Eq. (6).

The excellent fit for the velocity curves in Fig. 11 shows that Eq. (15) accurately describes the size dependence of the filament velocity. A plot of the dimensional filament velocity as function of the normalized size $\ell / \ell_{*}$ is presented in Fig. 13 for $c_{1}=0.76$ and $c_{2}=2.2$, which corresponds to $\Delta n / N=1$. In this case, the dimensional maximum velocity $V / V_{*}=0.33$ occurs for a filament size $\ell / \ell_{*}=0.41$. The normalized size for which the filament has maximum velocity as function of filament amplitude is presented in Fig. 14 and the corresponding filament velocity is presented in Fig. 15. It should be noted that $\ell_{*}$ over-estimates the cross-field size for which the filament moves fastest by more than a factor of two. Moreover, for relative filament amplitudes less than unity, $V_{*}$ over-estimates the maximum radial filament velocity by a factor of three or more.

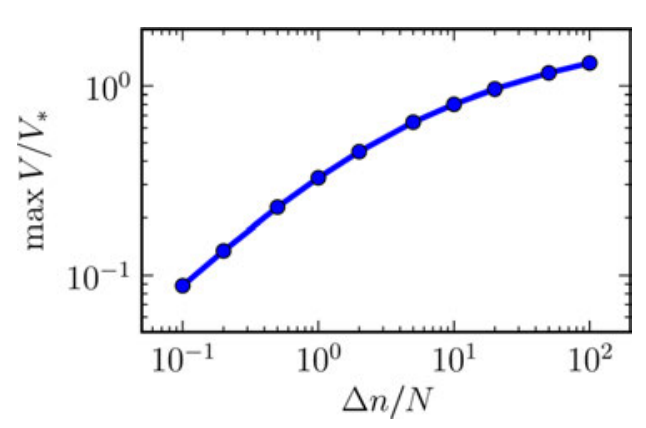

FIG. 15. (Color online) Maximum dimensional radial filament velocity as function of the relative filament amplitude in the presence of sheath dissipation. 


\section{CONCLUSIONS}

The interchange motion of field-aligned plasma filaments in non-uniformly magnetized plasmas has been investigated by means of numerical simulations. We have elucidated the filament velocity dependence on its amplitude and cross-field size. In the absence of sheath currents, or for weak sheath dissipation, the filament velocity increases with the square root of the cross-field size. For small amplitudes, the velocity increases as the square root of the relative amplitude. The velocity has a weak dependence on filament amplitude for relative amplitudes much larger than unity. The latter is the situation for the plasma blob experiments on the VTF device. ${ }^{24}$ Here, it was found that the filament velocity may approach the acoustic speed at low neutral gas pressures. The numerical simulations presented here are in favourable agreement with these experimental measurements, showing a persistent blob-like structure for the particle density and a dipole shape for the electrostatic potential. Comparison to these experimental measurements with inclusion of friction due to ion neutral collisions in the numerical simulations will be discussed in detail elsewhere.

Parallel currents through sheaths where the magnetic field lines intersect material walls result in damping of the collective motions on large spatial length scales. Thus, in the presence of sheath currents the filament velocity is largest for an intermediate cross-field structure size that depends on the magnetic field connection length. We have shown that the filament velocity dependence on its size according to a simple order of magnitude estimate is in excellent agreement with numerical simulations. This also explains the blob velocity scaling measured in the TORPEX experiment, where a scan of blob sizes was achieved by changing the ion mass. ${ }^{25}$

The application of a sheath dissipation term due to parallel currents in the model used in this and many other works assumes the filament structures to be perfectly elongated along the magnetic field. However, fluctuations and turbulence-driven transport in the scrape-off layer of tokamak plasmas is well know to be poloidally asymmetric due to regions of favourable and unfavourable magnetic field curvature. In order to take into account such ballooning effects, a three-dimensional model must be invoked where sheath boundary conditions are applied. An investigation of filament dynamics in such a geometry is now in progress.

${ }^{1}$ O. E. Garcia, Plasma Fusion Res. 4, 019 (2009).

${ }^{2}$ S. I. Krasheninnikov, D. A. D’Ippolito, and J. R. Myra, J. Plasma Phys. 74, 679 (2008).
${ }^{3}$ V. Naulin, J. Nucl. Mater. 363 365, 24 (2007).

${ }^{4}$ S. J. Zweben, J. A. Boedo, O. Grulke, C. Hidalgo, B. LaBombard, R. J. Maqueda, P. Scarin, and J. L. Terry, Plasma Phys. Controlled Fusion 49, S1 (2007).

${ }^{5}$ D. A. D’Ippolito, J. R. Myra, S. I. Krasheninnikov, G. Q. Yu, and A. Yu. Pigarov, Contrib. Plasma Phys. 44, 205 (2004).

${ }^{6}$ E. Sánchez, C. Hidalgo, D. López Bruna, I. García Cortés, R. Balbín, M. A. Pedrosa, B. van Milligen, C. Riccardi, G. Chiodini, J. Bleuel, M Endler, B. A. Carreras, and D. E. Newman, Phys. Plasmas 7, 1408 (2000).

${ }^{7}$ J. A. Boedo, D. L. Rudakov, R. A. Moyer, G. R. McKee, R. J. Colchin, M. J. Schaffer, P. G. Stangeby, W. P. West, S. L. Allen, T. E. Evans, R. J. Fonck, E. M. Hollmann, S. Krasheninnikov, A. W. Leonard, W. Nevins, M. A. Mahdavi, G. D. Porter, G. R. Tynan, D. G. Whyte, and X. Xu, Phys. Plasmas 10, 1670 (2003).

${ }^{8}$ G. Y. Antar, G. Counsell, Y. Yu, B. Labombard, and P. Devynck, Phys. Plasmas 10, 419 (2003).

${ }^{9}$ O. E. Garcia, J. Horacek, R. A. Pitts, A. H. Nielsen, W. Fundamenski, J. P. Graves, V. Naulin, and J. Juul Rasmussen, Plasma Phys. Controlled Fusion 48, L1 (2006).

${ }^{10}$ O. E. Garcia, J. Horacek, R. A. Pitts, A. H. Nielsen, W. Fundamenski, V. Naulin, and J. Juul Rasmussen, Nucl. Fusion 47, 667 (2007).

${ }^{11}$ O. E. Garcia, R. A. Pitts, J. Horacek, J. Madsen, V. Naulin, A. H. Nielsen, and J. Juul Rasmussen, Plasma Phys. Controlled Fusion 49, B47 (2007).

${ }^{12}$ O. E. Garcia, R. A. Pitts, J. Horacek, A. H. Nielsen, W. Fundamenski, J. P. Graves, V. Naulin, and J. Juul Rasmussen, J. Nucl. Mater. 363 365, 575 (2007).

${ }^{13}$ S. J. Zweben, D. P. Stotler, J. L. Terry, B. LaBombard, M. Greenwald, M. Muterspaugh, C. S. Pitcher, K. Hallatschek, R. J. Maqueda, B. Rogers, J. L. Lowrance, V. J. Mastrocola, and G. F. Renda, Phys. Plasmas 9, 1981 (2002).

${ }^{14}$ S. J. Zweben, R. J. Maqueda, D. P. Stotler, A. Keesee, J. Boedo, C. E. Bush, S. M. Kaye, B. LeBlanc, J. L. Lowrance, V. J. Mastrocola, R. Maingi, N. Nishino, G. Renda, D. W. Swain, J. B. Wilgen, and the NSTX Team, Nucl. Fusion 44, 134 (2004).

${ }^{15}$ J. L. Terry, N. P. Basse, I. Cziegler, M. Greenwald, O. Grulke, B. LaBom bard, S. J. Zweben, E. M. Edlund, J. W. Hughes, L. Lin, Y. Lin, M. Porko lab, M. Sampsell, B. Veto, and S. J. Wukitch, Nucl. Fusion 45, 1321 (2005).

${ }^{16}$ O. Grulke, J. L. Terry, B. LaBombard, and S. J. Zweben, Phys. Plasmas 13, 012306 (2006).

${ }^{17}$ M. Agostini, S. J. Zweben, R. Cavazzana, P. Scarin, G. Serianni, R. J. Maqueda, and D. P. Stotler, Phys. Plasmas 14, 102305 (2007).

${ }^{18}$ N. H. Bian, S. Benkadda, J. V. Paulsen, and O. E. Garcia, Phys. Plasmas 10, 671 (2003).

${ }^{19}$ O. E. Garcia, N. H. Bian, V. Naulin, A. H. Nielsen, and J. Juul Rasmussen, Phys. Plasmas 12, 090701 (2005).

${ }^{20}$ O. E. Garcia, N. H. Bian, and W. Fundamenski, Phys. Plasmas 13, 082309 (2006).

${ }^{21}$ S. I. Krasheninnikov, Phys. Lett. A 283, 368 (2001).

${ }^{22}$ D. A. D'Ippolito, J. R. Myra, and S. I. Krasheninnikov, Phys. Plasmas 9, 222 (2002).

${ }^{23}$ O. E. Garcia, N. H. Bian, V. Naulin, A. H. Nielsen, and J. Juul Rasmussen, Phys. Scr. T122, 104 (2006).

${ }^{24}$ N. Katz, J. Egedal, W. Fox, A. Le, and M. Porkolab, Phys. Rev. Lett. 101, 015003 (2008)

${ }^{25}$ C. Theiler, I. Furno, P. Ricci, A. Fasoli, B. Labit, S. H. Müller, and G. Plyushchev, Phys. Rev. Lett. 103, 065001 (2009).

${ }^{26}$ G. Q. Yu and S. I. Krasheninnikov, Phys. Plasmas 10, 4413 (2003).

${ }^{27}$ A. Y. Aydemir, Phys. Plasmas 12, 062503 (2005).

${ }^{28}$ J. R. Myra, D. A. D'Ippolito, D. P. Stotler, S. J. Zweben, B. P. LeBlanc, J. E. Menard, R. J. Maqueda, and J. Boedo, Phys. Plasmas 13, 092509 (2006). 